\title{
Técnicas para evaluar el potencial de comprensión y aprendizaje de los estudiantes
}

\author{
Karen D. Wood
}
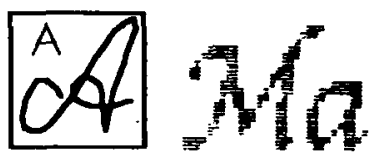

La mayoria de los profesores ha tenido alguna vez que vérselas con el problema de las desigualdades de sus alumnos en un tema tan central para la marcha de la clase como es el del nivel lector. La autora de este artículo propone en base a una revisión crítica de los procedimientos de evaluación existentes, un modo de evaluar que permite agrupar a los alumnos por sus dificultades y ajustar de este modo la acción pedagógica a esas dificultades.

El profesor suele ser consciente de que los resultados de sus alumnos varían notablemente según las exigencias concretas de cada tarea de lectura. Así, algunos alumnos comprenden mejor después de leer en silencio que en voz alta. Otros leen el material narrativo mejor que el material explicativo. $E$ incluso otros mejoran a ojos vistas cuando el profesor les proporciona conocimientos previos adicionales o preguntas exploratorias.

Dadas estas variaciones en la conducta de lectura de los alumnos, el uso de una única fuente de evaluación puede llevarnos a conclusiones erróneas sobre su potencial de aprendizaje. Es, pues, necesario evaluar la aptitud para la lectura bajo diversas circunstancias con objeto de determinar los niveles óptimos de rendimiento de los estudiantes.

El trabajo de Feuerstein (Feuerstein, Rand, y Joffman, 1979) destaca la importancia de determinar el potencial de aprendizaje de los estudiantes como parte del proceso de evaluación. Aunque sus esfuerzos estaban dirigidos a la evaluación de la inteligencia de individuos discapacitados, su filosofía es aplicable a la evaluación de la lectura.

Feuerstein contrasta su enfoque dinámico de la evaluación con aproximaciones estáticas que intentan cuantificar la conducta en términos de sim-

"Techniques for assessing students' potential for learning». The Reading Teacher, 1988, 41 (4), pp. 440-447. Reproducido con autorización de Karen D. Wood y de la International Reading Association (C). Traducción al castellano, CLEE, 1989 (Traducción de Carmen Trillo. La I.R.A. no se responsabiliza de la adecuación de la traducción). 
ples puntuaciones de test. Para implementar la evaluación dinámica, se requieren cuatro cambios:

1. El instrumento de evaluación debe ser reestructurado de forma que permita al examinador enseñar activamente al mismo tiempo que determina los efectos de esta enseñanza en la ejecución del examinando.

2. La situación de examen debe cambiar de un monólogo estandarizado y formateado a un diálogo que favorezca los intercambios orales y de interacción entre examinador y examinando.

3. El examinador debería buscar «los puntos más altos de ejecución» que llevan al examinando más allá del nivel inicial y permiten una demostración más amplia de capacidad.

4. El centro de interés del aprendizaje deberá estar más orientado al proceso que al producto.

\section{LA EVALUACION VARIADA ES MEJOR}

La evaluación dinámica puede ser considerada como una dimensión del cambio hacia una perspectiva más natural, en la que se observa cómo los estudiantes aportan su conocimiento inicial en los grupos de lectura, hacen inferencias y predicciones, y leen y relatan los sucesos de una selección (Moore, 1983). También se les observa en sus clases de contenido cuando tratan de determinar el significado de las palabras a partir del contexto, cuando responden tras la explicación del profesor, o se plantean preguntas.

Goodman (1985) se refiere a este proceso como «observación del niño" (kidwatching). Cunningham (1982) lo denomina simplemente «diagnóstico por observación» y Moore (1983) prefiere el término más global de «evaluación naturalista». En una situación de evaluación individual, el profesor/examinador utiliza diversas técnicas de intervención para proporcionar al alumno numerosas condiciones bajo las que demostrar su potencial adicional.

En los estudios realizados por Labov (1972) —que muestran que los individuos responden de forma diferencial ante la influencia de diferentes examinadores- encontramos más pruebas de la necesidad de diversificar las situaciones de evaluación para determinar el potencial de aprendizaje.

De igual modo, Harste y Carey (1979) lanzan la idea del «escenario de lo impreso" (print setting), destacando la importancia del contexto situacional en el que se encuentran los textos a la hora de desencadenar desde la memoria asociaciones específicas. La abundante investigación realizada sobre el contexto de lo impreso revela hasta qué punto puede alterarse la comprensión simplemente haciendo cambios en el contexto social (Baldwin y Readence, 1979; Carey, Harste, y Smith, 1981) o en las condiciones de aprendizaje (Goetz et al., 1983; Pichert y Anderson, 1977).

Este artículo utiliza los conceptos de evaluación dinámica y naturalista como principio fundamental para la modificación de los instrumentos utilizados en la toma de decisiones de clasificación. Mientras que Cioffi y Carney (1983) han demostrado la aplicabilidad del trabajo de Feuerstein a dispositivos estandarizados, este artículo mostrará su relevancia para instrumentos de evaluación informales, elaborados por el profesor.

Dado que la investigación indica que el libro de lectura básico se utiliza 
en más del $90 \%$ de las clases americanas en la actualidad (Intercambio de Información de Resultados Educativos, 1977; Wiesendanger y Birlem, 1981), mis ejemplos utilizan material del libro de lectura básico. Sin embargo, las técnicas son fácilmente adaptables a libros de biblioteca así como a las áreas de contenido.

\section{LA CLASIFICACION ADECUADA}

La preocupación diagnóstica más habitual en un profesor es determinar si los estudiantes están utilizando materiales adecuados a su capacidad lectora. Con frecuencia se utilizan al efecto los inventarios que acompañan a los programas básicos de lectura - normalmente una serie de listas de palabras graduadas y de textos con preguntas abiertas.

Además del hecho de que estos inventarios son dispositivos estáticos que dejan muy poco margen para una puntuación e interpretación flexibles, se plantean otros problemas a la hora de considerar su eficacia para clasificar a los alumnos. En primer lugar, normalmente se utiliza un pasaje de doscientas o más palabras para representar el nivel de los libros de cada curso, dando a los estudiantes una única oportunidad de demostrar competencia en ese nivel.

En segundo lugar, dado que los libros de lectura básicos contienen diversos géneros, que incluyen poesía, obras teatrales, historias y textos expositivos, un solo pasaje no permite que los estudiantes demuestren sus capacidades en todos los tipos de texto. En tercer lugar, la investigación ha demostrado que hay mucha variación intratexto en legibilidad en cuanto a libros de lectura básicos se refiere (Bradley y Ames, 1977).

Otros inventarios comerciales de construcción similar también son insuficientes para estos fines. Al igual que el inventario básico, los dispositivos comerciales normalmente utilizan un pasaje para representar el nivel de cada curso, pero los pasajes tienen poco que ver con los materiales utilizados en clase. Por tanto, su uso queda restringido a la evaluación general, como uno de los muchos dispositivos utilizados para representar la ejecución global del estudiante.

También es un error basarse en la puntuación de un test estandarizado y concluir que a un estudiante se le ha adjudicado correcta o incorrectamente los materiales de clase. Dichos tests no sólo nunca fueron concebidos para eso sino que fueron diseñados específicamente para comparar las puntuaciones de los estudiantes con las de la población con la que se normalizó el test (Farr y Carey, 1986).

Confiar tan sólo en el juicio de profesores anteriores también es inadecuado, dado que las capacidades de los estudiantes verían ante cambios de profesor y materia.

La «regla de oro» para determinar si la materia es apropiada para un determinado estudiante es simplemente "hacer que el estudiante lea ese contenido». Evaluar a los estudiantes en un medio distinto del que se les pide que trabajen podría denominarse "distanciar el test de la tarea».

Lo que se necesita por tanto es una aproximación estructurada para determinar cómo un individuo maneja la lectura de contenidos reales de libros de texto bajo condiciones que simulan la situación de la clase. Tal in- 
formación puede recogerse mediante la Matriz de Comprensión de Grupo y el Perfil de Comprensión Individual.

\section{LA MATRIZ DE COMPRENSION DE GRUPO}

Mientras que la evaluación «uno a uno» es necesaria con frecuencia para tomar decisiones sobre la correcta ubicación del alumno, no debería ser el único método utilizado. Dado que la mayor parte de la enseñanza de la lectura se organiza en pequeños grupos o círculos de lectura, un procedimiento válido sería determinar lo bien que determinados estudiantes funcionan en este contexto. Así los estudiantes son evaluados bajo condiciones establecidas por el profesor durante la actividad de lectura dirigida.

Una forma de organizar las observaciones recogidas en este tipo de evaluación es mediante la Matriz de Comprensión de Grupo (Wood, en prensa). Mediante esta matriz, el profesor puede concentrarse en el rendimiento del grupo completo o en el funcionamiento de un individuo en el contexto del grupo.

La matriz que aparece en la Figura 1 proporcioan una panorámica del grado de comprensión que un grupo de estudiantes manifiesta a partir de la historia «El Mandarín y El Mago» con la ayuda del profesor, según el modelo de Actividad Dirigida Lectura-Pensamiento de Stauffer (1969). Algunas categorías a observar son: el conocimiento previo de los estudiantes, sus capacidades de predicción e inferencia, su capacidad para expresar algo con sus propias palabras, y su capacidad para leer de forma uniforme y fluida.

Examinando la matriz de forma horizontal, aparece que los estudiantes del grupo analizado tienen dificultades para inferir información o para «leer entre líneas» y son incapaces de determinar los significados de las palabras a partir del contexto. Estos datos, junto con su insuficiente conocimiento previo, deberían sugerir inmediatamente al profesor que debe proporcionar información más detallada para ayudar a los estudiantes a visualizar el contexto y comprender el hilo de la historia.

Esta incapacidad para hacer inferencias y predicciones podría ser específica del relato, es decir, limitada únicamente a este texto. Sólo mediante futuras evaluaciones podría determinar el profesor si se ha evolucionado hacia una situación de carencia.

Examinando la matriz de forma vertical vemos que Jason es el que parece demostrar la mayor dificultad, aunque los comentarios del profesor indican que su evocación es buena y que su ejecución mejora en una situación de «uno a uno». Puede ser que la lectura delante de un grupo interfiera con su comprensión inferencial. Quizá en su caso el profesor decida utilizar una lectura silenciosa o un efoque conjunto de lectura, narración y discusión como alternativas para sesiones futuras.

Teresa parece ser algo impulsiva y puede necesitar ayuda para hacer inferencias y para controlar su propia comprensión.

Ryan acaba de entrar en este colegio el uno de octubre y, a juzgar por la calidad y superioridad de sus respuestas, puede necesitar que se le traslade al siguiente nivel. Sería útil una evaluación posterior con el Perfil de Comprensión individual para determinar la clasificación perfecta. 


\begin{tabular}{|c|c|c|}
\hline \multicolumn{2}{|c|}{ Retrato: El Mandarin y El Magos } & Fecha: 14 de octubre \\
\hline Género: & Narrativa (realista, fantasía) & Curso: $4 .^{\circ}$ \\
\hline & Poesia & \\
\hline & Teatro & \\
\hline & Exposición & \\
\hline
\end{tabular}

Hace predicciones sobre el relato

Participa en la discusión

Contesta preguntas de todos los niveles

Determina los significados de las palabras mediante el contexto

Lee de forma fluida $y$ uniforme

Puede contar el texto con sus propias palabras

Comprende tras lectura silenciosa

Puede leer *entre líneas*

Posee amplio conocimiento previo

\section{* Nuevo estudiante-1 de octubre}

\begin{tabular}{|c|c|c|c|c|c|c|}
\hline$\stackrel{2}{y}$ & $\underset{\widetilde{\pi}}{\stackrel{5}{x}}$ & $\sum^{\bar{E}}$ & 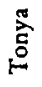 & 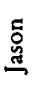 & 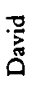 & 总 \\
\hline$S$ & + & - & $S$ & - & + & - \\
\hline$S$ & + & $S$ & $S$ & - & + & $S$ \\
\hline- & + & $S$ & $S$ & $S$ & + & $S$ \\
\hline- & + & - & - & - & $S$ & - \\
\hline+ & + & + & $S$ & - & + & $S$ \\
\hline$S$ & + & + & $S$ & $S$ & $S$ & - \\
\hline$N$ & $N$ & $N$ & $N$ & $N$ & $N$ & $N$ \\
\hline- & $S$ & $S$ & - & - & $S$ & - \\
\hline$S$ & $S$ & - & - & - & $S$ & - \\
\hline
\end{tabular}

Comentarios: Los estudiantes tenian gran dificultad en comprender la bistoria basta que les proporcioné mucha más información previa. Sus predicciones ni fueron tan precisas ni abundantes como de costumbre -en gran parte debido a su falta de conocimiento de las dinastias chinas. Jason se queda muy callado a menos que se le hagan preguntas concretas. Reacciona con más entusiasmo al uno a uno. Mientras que su evocación es buena, su lectura oral es muy entrecortada. Teresa siempre se ofrece voluntaria para responder, aunque su evocación se encuentra en el nivel literal. Ryan puede necesitar que se le traslade a otro nivel - se le evaluará individualmente.

$\begin{array}{lcc}\text { A menudo } & + & \text { Palabras para revisar: } \\ \text { Algunas veces } & \mathrm{S} & \frac{\text { dinastia }}{\text { Mandarin }} \\ \text { Raramente } & - & \text { cola } \\ \text { No se observa } & \mathrm{N} & \end{array}$


La matriz de Comprensión de Grupo no debería utilizarse más de una vez al mes, aunque el profesor observador puede seguir tomando notas mentales considerando los puntos fuertes y débiles de los estudiantes a través de todas las sesiones de lectura. Al igual que con el Perfil de Comprensión Individual que describimos a continuación, la ejecución de los estudiantes debería ser analizada utilizando diferentes tipos de prosa.

Se pueden elaborar matrices similares que den una idea general de las tareas comunes a las diversas áreas de çontenido. Además de las categorías listadas en la Figura 1, otras capacidades alternativas observadas podrían ser: «asimila nuevo vocabulario antes de que se le enseñe», "controla su propia comprensión", "ofrece conocimiento previo», "completa satisfactoriamente las tareas del libro de texto", por citar unas cuantas.

\section{EL PERFIL DE COMPRENSION INDIVIDUAL}

El Perfil de Comprensión Individual ha sido modificado y adaptado para su uso en clase a partir del propuesto por Cunningham et al. (1983). Como puede verse en la Figura 2, la matriz es un dispositivo elaborado por el profesor para organizar y determinar modelos de conducta y lectura bajo diversas condiciones.

Para averiguar el rendimiento de un alumno le pedimos que lea directamente el libro de lectura básico bajo unas condiciones que se han modificado para coordinar con las tareas esperadas en la clase. Las cuatro condiciones evaluadas son listadas de izquierda a derecha en la parte superior de la matriz y se describen a continuación.

\section{Tipo de lectura}

Se debería permitir a los estudiantes que demostrasen tanto sus capacidades para la lectura oral como silenciosa. Muchos estudiantes son más competentes en una modalidad.

\section{Géneros}

Dado que muchos estudiantes pueden mostrar una preferencia hacia una forma determinada de literatura en términos de interés, capacidad o de ambos, la evaluación debería incluir ejemplos que representen los diversos contenidos del texto. Además de aparecer aquí asociado a un libro de lectura básico, el formato también es apropiado para materiales específicos de área. Categorías sugeridas para un texto de estudios sociales podrian ser comparación y contraste, causa y efecto, vocabulario en contexto, interpretación de cuadros, mapas y gráficos por mencionar algunas.

\section{Modalidad de evocación}

Medidas de narración libre o registrada, proporcionan a los estudiantes más «libertad para evocar" (Wood, 1985), permitiéndoles utilizar sus propias experiencias para conceder importancia a la información textual. Los estudiantes no tienen que responder adivinando lo que piensa el profesor, sino que pueden asociar la información en cualquier orden y demostrar un margen de conocimiento más amplio. 
FIGURA 2

El Perfil de Comprensión Individual

\begin{tabular}{|c|c|c|c|c|c|c|c|c|c|c|c|c|c|c|c|}
\hline & \multicolumn{2}{|c|}{$\begin{array}{l}\text { Tipo de } \\
\text { lectura }\end{array}$} & \multicolumn{5}{|c|}{ Género } & \multicolumn{3}{|c|}{$\begin{array}{l}\text { Tipo de } \\
\text { memorización }\end{array}$} & \multicolumn{3}{|c|}{$\begin{array}{l}\text { Grado de } \\
\text { orientación }\end{array}$} & \multirow{2}{*}{$\begin{array}{c}\begin{array}{c}\text { Comprens. } \\
\text { global }\end{array} \\
\\
\\
1=\text { nada } \\
2=\text { algo } \\
3=\text { la } \\
\text { mayor } \\
\text { parte } \\
4=\text { todo }\end{array}$} & \multirow[t]{2}{*}{ Comentarios } \\
\hline & 팽 & 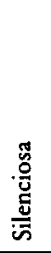 & 墨 & 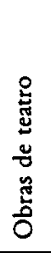 & 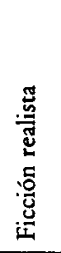 & 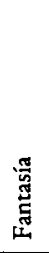 & 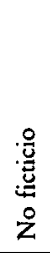 & 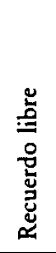 & 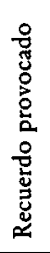 & 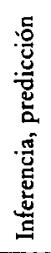 & 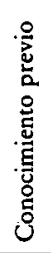 & 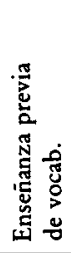 & 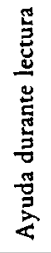 & & \\
\hline $\begin{array}{l}\text { Nivel } 22 \\
\text { p. } 41\end{array}$ & $\sim$ & & & & r & & & $r$ & r & & - & - & - & 3 & Un poco entrecortado al principio; después muy fluido con evocación precisa \\
\hline $\begin{array}{l}\text { Nivel } 22_{2} \\
\text { p. } 76\end{array}$ & $\sim$ & & & 2 & & & & $r$ & r & $\sim$ & - & - & - & 4 & Lectura y narración muy fluidas \\
\hline $\begin{array}{l}\text { Nivel } 22_{2} \\
\text { p. } 168\end{array}$ & & $r$ & & & & & 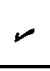 & $\boldsymbol{r}$ & 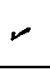 & $r$ & - & - & - & 4 & No necesita ayuda - tiene control del reconocimiento y comprensión de las palabras \\
\hline $\begin{array}{l}\text { Nivel } 3, \\
\text { p. } 101\end{array}$ & r & & & & $r$ & & & $r$ & $\boldsymbol{r}$ & & - & - & - & 2 & $\begin{array}{l}\text { Algunos problemas de fluidez y recuerdo impreciso (p.e., dijo «estela* por *intento* } \\
\text { y *hermoso* por *cuerpo*. (En inglés, *trail por *trial» y *beautiful por *body*.) }\end{array}$ \\
\hline $\begin{array}{l}\text { Nivel 3, } \\
\text { p. } 96\end{array}$ & $r$ & & & & & $\sim$ & & $\boldsymbol{r}$ & $\boldsymbol{r}$ & 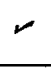 & $\sim$ & $\sim$ & & 3 & Con ayuda, la evocación mejora; puede predecir e inferir (p.e., ¿por qué crees que...) \\
\hline $\begin{array}{l}\text { Nivel } 3, \\
\text { p. } 66\end{array}$ & & $\boldsymbol{r}$ & & & & & $r$ & $\boldsymbol{r}$ & $\sim$ & & $\boldsymbol{r}$ & r & 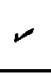 & 4 & 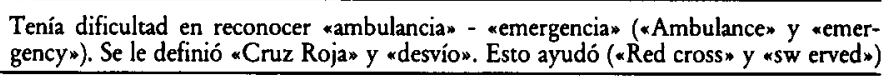 \\
\hline $\begin{array}{l}\text { Nivel } 31 \\
\text { p. } 119\end{array}$ & & $r$ & & & & & 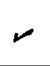 & $r$ & $\boldsymbol{r}$ & $\sim$ & $\boldsymbol{r}$ & レ & & 4 & Probó con y sin ayuda La comprensión mejoró con ayuda. \\
\hline
\end{tabular}

Evaluación global: La comprensión de Eric en lectura silenciosa parece mejor que en lectura oral. Puede expresarse con sus propias palabras en el nivel 3 , pero da más detalles cuando se le pregunta o sugiere. Con ayuda, parece beneficiarse de la enseñanza con este material. 
Se prefiere esto a pedir a los estudiantes que contesten una lista de preguntas abiertas, reconozcan la alternativa correcta, o rellenen una palabra o frase omitidas. Además, las capacidades de los estudiantes para inferir los elementos que faltan y predecir resultados lógicos pueden evaluarse mediante este procedimiento de interrogación sugerida.

\section{Grado de orientación}

Atenerse estrictamente a las indicaciones de administración de los inventarios comerciales significa que el examinador no puede ayudar al examinando de ninguna manera. Este enfoque estático falla a la hora de ilustrar el proceso dinámico y en continuo cambio de la comprensión.

Al utilizar la evaluación dinámica, el profesor debería probar únicamente distintos métodos para determinar cómo aprende mejor un estudiante. Por ejemplo, si un estudiante muestra problemas de fluidez y recuerdo impreciso de un texto, el profesor puede enseñar en primer lugar las palabras que representan obstáculos significativos y establecer una conexión con el conocimiento previo relevante para ver si los problemas se mitigan.

También el profesor puede proporcionar ayuda durante la lectura para determinar qué estrategias metacognitivas posee el estudiante para lograr el significado y la coherencia. Dicha ayuda puede incluir preguntar al estudiante si lo que acaba de leer tiene sentido y, si no lo tiene, qué palabra o palabras están poco claras; o puede incluir el enseñar al estudiante que siga leyendo para determinar el significado o pronunciación de una palabra desconocida.

Conjuntamente con estas ideas sobre lectura, el profesor debería preguntarse continuamente: «¿está comprendiendo el estudiante?» Lo que puede determinar utilizando una escala como la siguiente:

\section{Comprensión global:}

No comprende

Comprende algunas veces..................................................... 2

Comprende la mayor parte de las veces .................................... $\quad 3$

Comprende todo el tiempo ....................................................... 4

Como muestra la Figura 2, la lectura de Eric era entrecortada al principio, lo que daba a entender un posible malestar en la situación de evaluación. Sin embargo, tras unos pocos intentos era evidente que tenía un completo control del vocabulario y de la comprensión y que no necesitaba la ayuda del profesor.

En el nivel 3, tenía dificultad en reconocer algunas de las palabras. Aparentemente, algunas no estaban en su vocabulario de lectura, aunque sí se encontraban en su vocabulario de escucha (p.e, intento, hermoso, ambulancia, emergencia). Otras palabras (p.e., Cruz Roja, desvío), cuando se le enseñaban en un contexto significativo, oral, se le hacían claras, lo que ayudaba a su narración posterior. Por consiguiente, el profesor concluyó que, con orientación adecuada, Eric podría beneficiarse de la enseñanza del libro de lectura básico del nivel $3_{1}$. 
En general, es mejor comenzar en el nivel básico inferior al que se espera que esté el estudiante y avanzar hacia un nivel superior en la medida en que sea necesario. Esto permite que el estudiante experimente el éxito al principio y disminuya la ansiedad de la evaluación. Por regla general, un profesor intuitivo sabrá después de pocas lecturas si se necesita avanzar o retroceder en los materiales.

Tras algunas experiencias con el Perfil de Comprensión Individual, disminuye el tiempo de administración, dado que los profesores se familiarizan más con los puntos fuertes y débiles de los estudiantes. En consecuencia, este tipo de dispositivo de clasificación es más informativo y necesita menos tiempo que el inventario de lectura informal.

\section{UNA NOTA FINAL}

La evaluación con fines de clasificación no debería ser un episodio que tiene lugar una vez al año cuando comienza el colegio. La evaluación en una sola vez da como resultado «un etiquetado básico» (Basal labeling) y "un seguimiento básico» (Basal tracking), donde la clasificación actual se determina mediante la del año anterior, y el progreso se determina mediante la evaluación intermitente de habilidades (Wood, 1984). Lo que queda sin explorar en tal enfoque es hasta qué punto pueden comprender los estudiantes los textos reales del libro de lectura básico. En consecuencia, los estudiantes pueden estar etiquetados permanentemente en el grupo de lectura inferior y se les puede seguir a través del libro de lectura básico sin reconocer las posibles mejoras en el rendimiento.

Con las técnicas descritas en este artículo la evaluación se considera como un proceso dinámico y contínuo diseñado para evaluar el potencial de aprendizaje de los estudiantes en condiciones naturales. De este modo, estas técnicas evalúan a los estudiantes en el acto de la comprensión y al profesor en el acto de enseñar.

\section{Referencias}

BALDWIN, R. SCOTT y JOHN E. READENCE. «Critical Reading and Perceived Authority». Journal of Reading, vol. 22 (abril de 1979), pp. 617-22.

BRADLEY, JOHN M. y WILBUR, S. AMES. «Readability Parameters of Basal Readers». Journal of Reading Bebavior, vol. 9 (verano de 1977), pp. 175-83.

CAREY, ROBERT F.; JEROME, C.; HARSTE y SHARON, L. SMrTH. «Contextual Constraints and Discourse Processes: A Replication Study». Reading Research Quarterly, vol. 16, n. 2 (1981), pp. 201-12.

Cioffi, Grant y John J. CARney. «Dynamic Assessment of Reading Disabilities». The Reading Teacher, vol. 36 (abril de 1983), pp. 764-75.

CunNINGHAM, PATRICIA M. "Diagnosis by Observation". En Approaches to the Informal Evaluation of Reading. Editado por John J. Pikulski y Timothy Shanahan. Newark, DE: International Reading Association, 1982.

Cunningham, Patricia M.; Sharon, Arthur Moore; James W. Cunningham y David W. MOORE. Reading in Elementary Classrooms: Strategies and Observations. Nueva York, NY: Longman, 1983.

Education Products Information Exchange. Report on a National Study of the Quality of Instructional Materials Most Used by Teachers and Learners. Technical Report, n. $0^{\circ}$. Nueva York, NY. EPIE Institute, 1977.

FARR, ROGER y ROBERT F. CAREY. Reading: What Can Be Measured? Newark, DE: International Reading Association, 1986. 
Feuerstein, Reuven; YAacov, Rand y Mildred B. Hoffman. The Dynamic Assessment of Retarded Performance. Baltimore, MD: University Park Press, 1979.

GoETZ, ERNEST T.; DiANE L. SCHALleRT, RALPH E. ReYNOLDS y DEAN I. RADIN. «Reading in Perspective: What Real Cops and Pretend Burglars Look for in a Story*. Joumal of Educational Psychology, vol. 75 (agosto de 1983), pp. 500-610.

GOODMAN YETTA, M. *Kidwatching Observing Children in the Classroom*. En Observing the Language Learner. Editado por Angela Jaggar y M. Trika Smith-Burke. Newark, DE: International Reading Association, 1985.

Harste Jerome C. y Robert F. CareY. «Comprehension as Setting*. En Monograph in Language and Reading Studies: New Perspectives on Comprebension. Editado por Jerome C. Harste y Robert F. Carey. Bloomington, IN: Indiana University School of Education, 1979 .

LABOV, WILlIaM. Language in the Inner City. Philadelphia, PA: University of Pennsylvania Press, 1972.

MOORE, DAVID W. «A Case for Naturalistic Assessment of Reading Comprehension». Language Arts, vol. 60 (noviembre-diciembre de 1983), pp. 957-69.

Pichert, James W. y Richard, C. Anderson. "Taking Different Perspectives on a Story». Joumal of Educational Psychology, vol. 69 (agosto de 1977), pp. 309-15).

StTAuffer, Russell G. Directing Reading Maturity as a Cognitive Process. Nueva York, NY: Harper and Row, 1969.

WIESENDANGER, KATHERINE y ELLEN BIRLEM. *A Critical Look at the Reading Approaches and Grouping Patterns Currently Used in the Primary Grades». Reading Horizons, vol. 22 (otoño de 1981), pp. 54-58.

WOOD, KAREN D. «Evaluating Progress in the Language Arts». En Language Arts Instruction and the Beginning Teacher: A Practical Guide. Editado por Carl R. Personke y Dale D. Johnson. Englewood Cliffs, NJ: Prentice Hall, en prensa.

Wood, Karen D. *Free Associational Assessment: An Alternative to Traditional Testing». Journal of Reading, vol. 29 (noviembre de 1985), pp. 106-11.

WOOD, KAREN D. «Read First. Test Later: Meeting the Needs of the "Overskilled Reader"». Reading Horizons, vol. 24 (invierno de 1984), pp. 133-40.

\section{Datos sobre la autora}

Wood es Cordinadora de los Programas de Educación en Lectura del nivel medio en el Departamento de Curriculum e Instrucción en la Universidad de Carolina del Norte, en Charlotte. Está particularmente interesada en aplicar la investigación y la teoria a la práctica escolar. 